University of Wollongong

Research Online

Australian Institute for Innovative Materials -

Papers

Australian Institute for Innovative Materials

$1-1-2015$

Porous AgPd-Pd composite nanotubes as highly efficient electrocatalysts for lithium-oxygen batteries

Wenbin Luo

University of Wollongong, w1368@uowmail.edu.au

Xuanwen Gao

University Of Wollongong, xg973@uowmail.edu.au

Shulei Chou

University of Wollongong, shulei@uow.edu.au

Jiazhao Wang

University of Wollongong, jiazhao@uow.edu.au

Hua-Kun Liu

University of Wollongong, hua@uow.edu.au

Follow this and additional works at: https://ro.uow.edu.au/aiimpapers

Part of the Engineering Commons, and the Physical Sciences and Mathematics Commons

Research Online is the open access institutional repository for the University of Wollongong. For further information contact the UOW Library: research-pubs@uow.edu.au 


\title{
Porous AgPd-Pd composite nanotubes as highly efficient electrocatalysts for lithium-oxygen batteries
}

\author{
Abstract \\ Porous AgPd-Pd composite nanotubes (NTs) are used as an efficient bifunctional catalyst for the oxygen \\ reduction and evolution reactions in lithium-oxygen batteries. The porous NT structure can facilitate rapid \\ $\mathrm{O} 2$ and electrolyte diffusion through the NTs and provide abundant catalytic sites, forming a continuous \\ conductive network throughout the entire energy conversion process, with excellent cycling performance.

\section{Keywords} \\ agpd, pd, composite, nanotubes, highly, efficient, porous, electrocatalysts, batteries, lithium, oxygen \\ Disciplines \\ Engineering | Physical Sciences and Mathematics

\section{Publication Details} \\ Luo, W., Gao, X., Chou, S., Wang, J. \& Liu, H. (2015). Porous AgPd-Pd composite nanotubes as highly \\ efficient electrocatalysts for lithium-oxygen batteries. Advanced Materials, 27 (43), 6862-6869.
}




\title{
Porous AgPd-Pd Composite Nanotubes as Highly Efficient Electrocatalysts for Lithium-Oxygen Batteries
}

\author{
Wen-Bin Luo, Xuan-Wen Gao, Shu-Lei Chou, *Jia-Zhao Wang, and Hua-Kun Liu
}

Porous nanotubes (NTs) have become increasingly important nanomaterials in electronics, energy storage, catalysis, and fuel cell applications. ${ }^{[1-7]}$ In contrast to the intact walls of conventional NTs, this structural feature will result in a much more adsorption efficiency and abundant active catalytic sites, because molecules and electrolyte can enter into the hollow cavities of porous NTs via not only the two narrow ends but also holes along the tube wall..$^{[3,7-9]}$ Especially in the field of energy storage and conversion, these $1 \mathrm{D}$ porous nanostructures can also form a continuous conductive network and improve the adsorption of and immersion in electrolyte on the surfaces of electroactive materials in order to facilitate the electrode reaction kinetics for high energy density. ${ }^{[10-14]}$ This porous $1 \mathrm{D}$ structure will be even more promising for increasing the catalytic activities toward the two key processes in lithium oxygen battery, oxygen reduction reaction (ORR) $\left(\mathrm{O}_{2}+2 \mathrm{Li}^{+}+2 \mathrm{e}^{-} \rightarrow \mathrm{Li}_{2} \mathrm{O}_{2}\right)$ and the oxygen evolution reaction (OER) $\left(\mathrm{Li}_{2} \mathrm{O}_{2} \rightarrow \mathrm{O}_{2}+2 \mathrm{Li}^{+}+2 \mathrm{e}^{-}\right)$by facilitating rapid $\mathrm{O}_{2}$ diffusion and electrolyte accessibility, and providing more catalytic reaction sites for deposition of $\mathrm{Li}_{2} \mathrm{O}_{2} \cdot{ }^{[15-20]}$ More importantly, this 1D nanostructured catalyst may solve many of the inherent catalytic problems associated with stateof-the-art nanoparticulate catalysts. ${ }^{[21-23]}$ The porous NTs are characterized by their uniquely anisotropic nature, which offers advantageous structural and electronic factors to the catalytic reduction of oxygen. ${ }^{[13]}$ The galvanic replacement method provides a simple and versatile route for producing these hollow nanostructures in composite form with controllable pore structures. ${ }^{[24-26]}$ A number of metals with NT structure have been successfully synthesized by using silver or copper nanowire as templates, such as Pt, Pd, and Au NTs. ${ }^{[25-27]}$ As compared to pure monometallic systems, bimetallic catalysts have further garnered considerable interest because they exhibit distinctly different and often superior activity toward many chemical transformations, as determined via density functional theory calculations and experimental studies. ${ }^{[28-35]}$ Because of a combination of ligand, geometric, and/or ensemble effects, bimetallic catalysts strongly enhance the kinetics of the ORR and OER. $[8,21,28,32,36-39]$ Therefore, some bimetallic composites with NT structure have been further designed by controlling the reaction process. For example, Ag nanowires coated with AgPd alloy sheaths were synthesized and used for reversible

W.-B. Luo, Dr. X.-W. Gao, Dr. S. L. Chou,

Prof. J.-Z. Wang, Prof. H.-K. Liu

Institute for Superconducting and Electronic Materials

University of Wollongong

Wollongong, NSW 2522, Australia

E-mail: shulei@uow.edu.au

DOI: 10.1002/adma.201502262 absorption and desorption of hydrogen. ${ }^{[40]}$ The AgPd alloy sheaths act as a highly efficient catalyst during the reversible absorption and desorption of hydrogen, owing to the combination of geometric and synergistic effects. ${ }^{[38-41]}$ To the best of our knowledge, however, there is still no report on using porous AgPd NTs as electrocatalyst for $\mathrm{Li}-\mathrm{O}_{2}$ batteries.

Herein, we use the galvanic replacement reaction to synthesize 1D AgPd-Pd composite porous NTs, which can act as an efficient bifunctional catalyst for the ORR and OER in lithiumoxygen batteries. This porous NT structure shows favorable rechargeability and excellent energy efficiency, facilitating rapid $\mathrm{O}_{2}$ and electrolyte diffusion through the NTs, as well as forming a continuous conductive network throughout the whole energy conversion process.

The electrocatalysts with NT structure were synthesized via a galvanic replacement reaction of a concentration controlled $\mathrm{Pd}^{2+}$ solution and silver nanowire $(\mathrm{Ag} \mathrm{NW}$ ) templates (see the Experimental Section for more details). The growth of the NTs was closely monitored during the synthesis under different conditions and characterized by a combination of X-ray diffraction (XRD), field emission scanning electron microscopy (FESEM), and selected area electron diffraction (SAED) of individual NTs, as shown in Figure 1 and Figure S1 (Supporting Information). The 1D Ag NWs exhibit a range of diameters around $100 \mathrm{~nm}$ and pure face-centered-cubic (fcc) phase, as shown in Figure 1a and Figure S1(a1) (Supporting Information). The FESEM image of the Ag NWs shows pentagonal cross-sections (Figure S1(a1), Supporting Information). According to previously reported results, ${ }^{[25,42]}$ the nanowire structure growing along the [111] direction can be interpreted as a chain of decahedra joined along the vertex in parallel to the fivefold symmetry. The unique fivefold symmetry is due to the existence of five twin planes that cross along a line in the center of each nanowire. The same results can also be confirmed by the SAED pattern in our work from the overlapping [001] and [112] zone axes, as shown in Figure S1(a2) (Supporting Information). By refluxing the $\mathrm{Ag}$ NWs with a controlled aqueous $\mathrm{Pd}^{2+}$ solution, NT-like structures were formed at different replacement reaction depths, marked as AgPd-1, AgPd-2, and AgPd-3 NTs in this Communication. In Figure $1 \mathrm{c}, \mathrm{d}$, compared with pure Ag NWs, three obvious peaks at $40.1^{\circ}, 46.6^{\circ}$, and $68.1^{\circ}$ gradually appear in the AgPd-2 and AgPd-3 NT composites, owing to the galvanic replacement reaction between the $\mathrm{Ag} / \mathrm{Ag}^{+}$and $\mathrm{Pd} / \mathrm{Pd}^{2+}$ pairs, corresponding to the (111), (200), and (220) crystalline planes of Pd. Meanwhile, the Ag reflection peaks, from the (111), (200), and (220) planes, shift right with increasing Pd reaction weight ratio from Figure $1 \mathrm{~b}$ to Figure $1 \mathrm{~d}$. Since AgPd alloy has a smaller lattice constant than Ag, the shift of the reflections can be attributed to the formation of AgPd alloy. Therefore, the diffraction patterns can be indexed as mixed phase AgPd alloy 


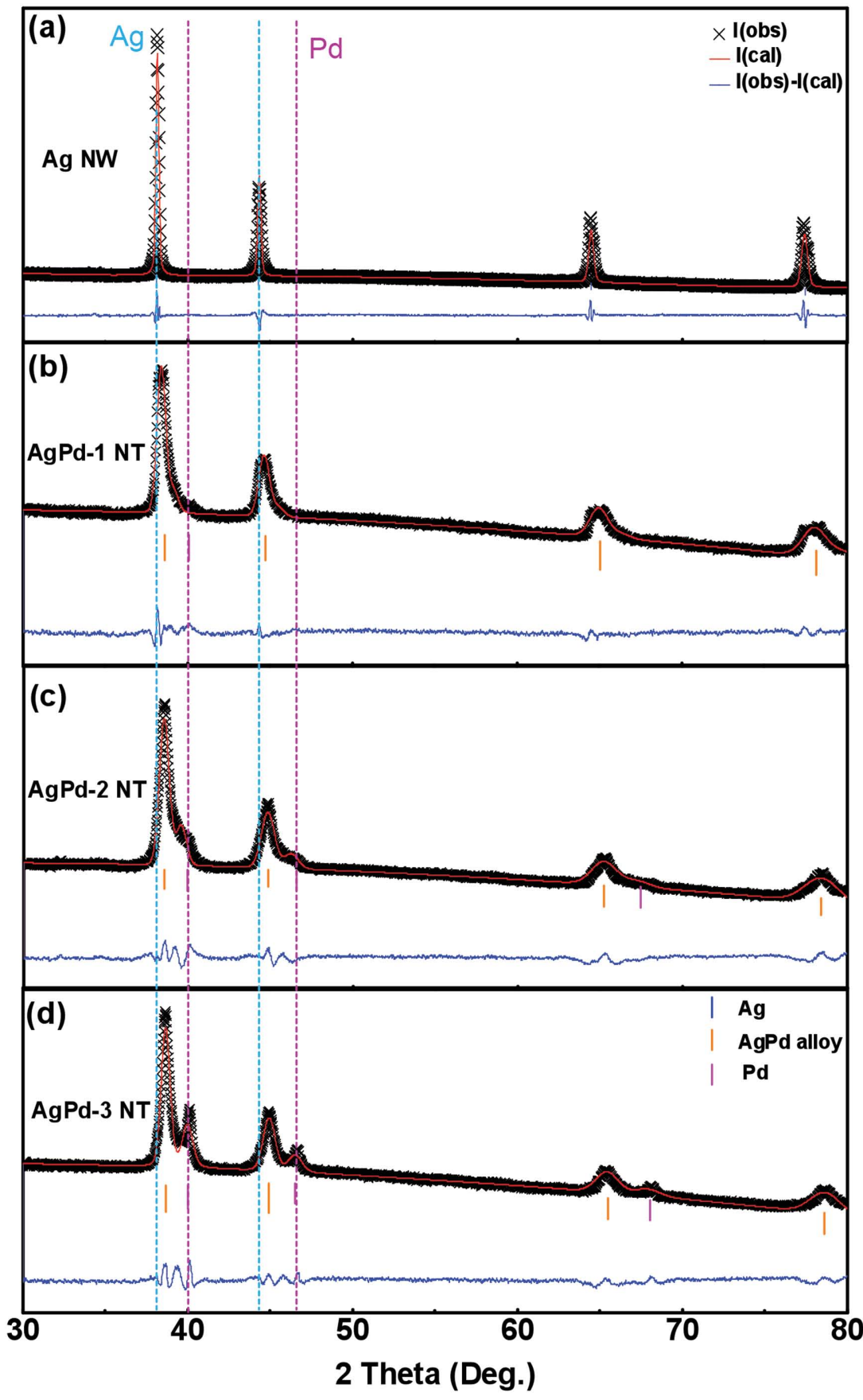

Figure 1. XRD Rietveld refinement results for a) Ag NWs, and b) AgPd-1, c) AgPd-2, and d) AgPd-3 NTs.

and Pd, as indexed in the XRD Rietveld refinement results in Figure 1a-d. The details of phase fractions and unit cell parameters are displayed in Table S1 (Supporting Information). The unit cell parameter and cell volume show a decreasing trend with the depth of the galvanic replacement reaction between $\mathrm{Ag}$ and Pd. AgPd alloy begins to form during the replacement reaction. This conclusion is also consistent with the previous results reported by Sastry and co-workers. ${ }^{[43]}$ The AgPd alloy phase fraction in AgPd-1 is 83\%, and the alloy content experiences a decrease down to $70 \%$ in AgPd-3. Meanwhile, the Pd phase fraction increases from $17 \%$ in AgPd-1 to 30\% in AgPd-3. The ratio of Ag to Pd in AgPd alloy also falls from 9:1 in AgPd-1 to 1:1 in AgPd-3. As shown in the FESEM images (Figure S1(c1,d1), Supporting Information), the as-prepared NTs still retain the same fivefold cross-sectional structure as the pure silver in terms of morphology. This is also indicated by the SAED patterns (Figure S1(c2,d2), Supporting Information), with overlapping of [001] and [112] zone axes in the fcc unit cell.

Schematic diagram of the formation of the NTs and corresponding transmission electron microscope (TEM) images are shown in Figure 2. TEM images in Figure 2b-e show that the as-prepared $\mathrm{Ag} \mathrm{NWs}$ and $\mathrm{AgPd}$ composites have similar 1D structured morphology. A schematic illustration of the galvanic replacement reaction process is also presented in Figure 2a. A series of AgPd NTs (AgPd-1, AgPd-2, and AgPd-3) with different morphologies and different replacement depths can be obtained. The replacement reaction occurs because the reduction potential of $\mathrm{Pd}^{2+} / \mathrm{Pd}$ is higher than that of $\mathrm{Ag}^{+} / \mathrm{Ag}$. $\mathrm{Pd}^{2+}$ ions diffuse onto the surfaces of the $\mathrm{Ag}$ templates, and then oxidize $\mathrm{Ag}$ to $\mathrm{Ag}^{+}$. The released electrons can easily move to the surfaces of the templates and reduce $\mathrm{Pd}^{2+}$ to $\mathrm{Pd}$, leading to the formation of a thin layer of AgPd alloy. ${ }^{[13,24,40]}$ In this reaction process, all the atomic diffusion and replacement will cause structural redistribution, to form some hollow (red circle marked in Figure 2c) or even porous NT structures (inset image in Figure 2e). This process is clearly indicated by the energy dispersive X-ray spectroscopy (EDS) with scanning transmission electron microscopy (STEM) in a highly resolved small area of $250 \mathrm{~nm}$ and even $20 \mathrm{~nm}$ on the walls of the NTs. In the AgPd-1 NT composite, Pd and $\mathrm{Ag}$ show a similar distribution over the entire area to all the other AgPd samples. With Ag being oxidized, in the AgPd-3 NT composite, the Pd signal has a higher distribution than $\mathrm{Ag}$ on the surface of the NTs (Figure S2, Supporting Information). The change in the crystalline plane distance on the wall of the NT also indicates the increasing amount of Pd. In the AgPd-1 NT composite (Figure 2c2), the lattice fringes have a measured interplanar distance of $1.98 \AA$, smaller than $2.04 \AA$ for the fcc Ag (200) and higher than $1.94 \AA$ for the fcc Pd (200), which can be indexed to the (200) crystalline plane of AgPd alloy. Compared with the AgPd-1 NTs, the lattice fringes from the wall in AgPd-3 NT composite have measured distances of 2.25 and $1.36 \AA$ (Figure 2e2), which can be indexed to the (111) and (220) planes of fcc Pd, respectively, confirming that Pd 

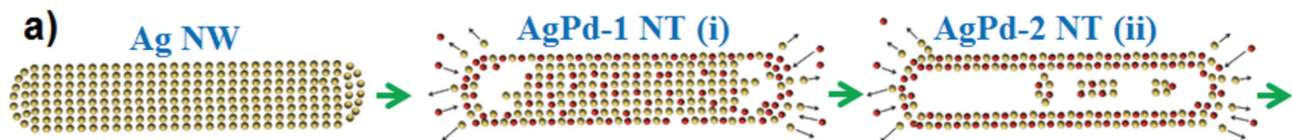

www.MaterialsViews.com

b)
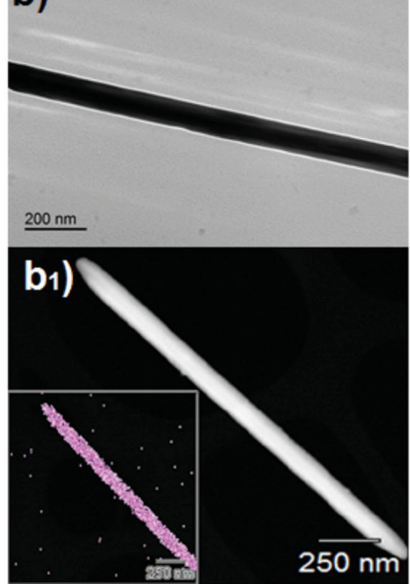

b2)

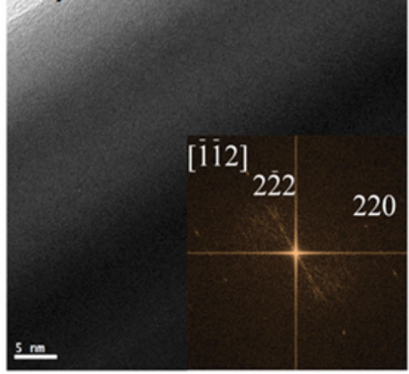

c)

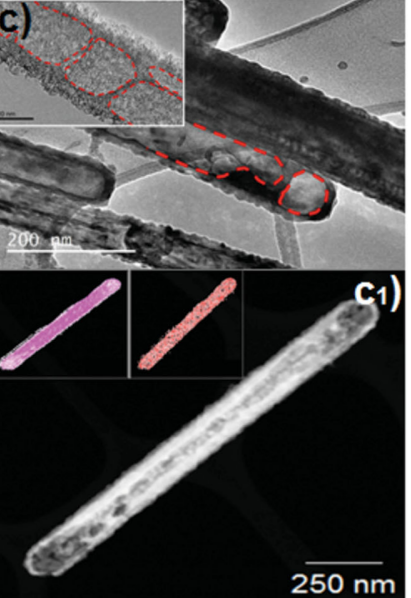

c)

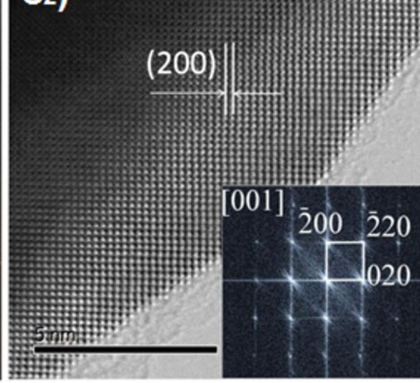

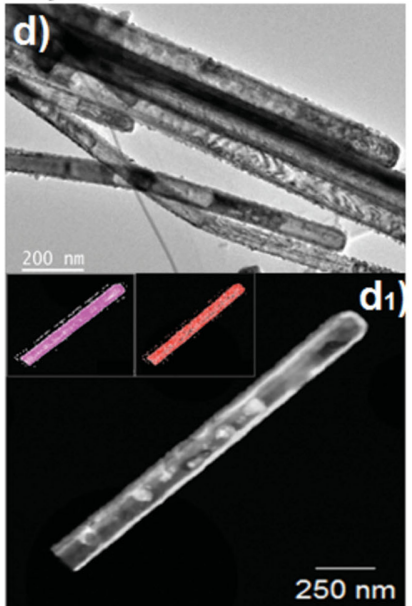

d2)

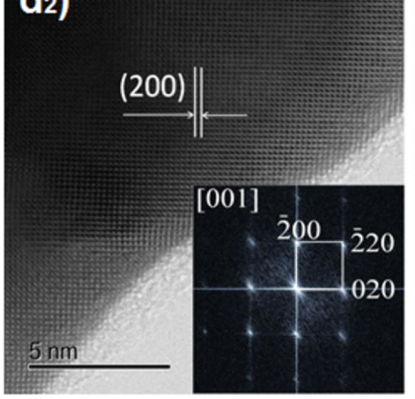

AgPd-3 NT (iii)

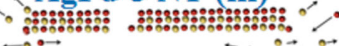
$\because \because \because$ 280
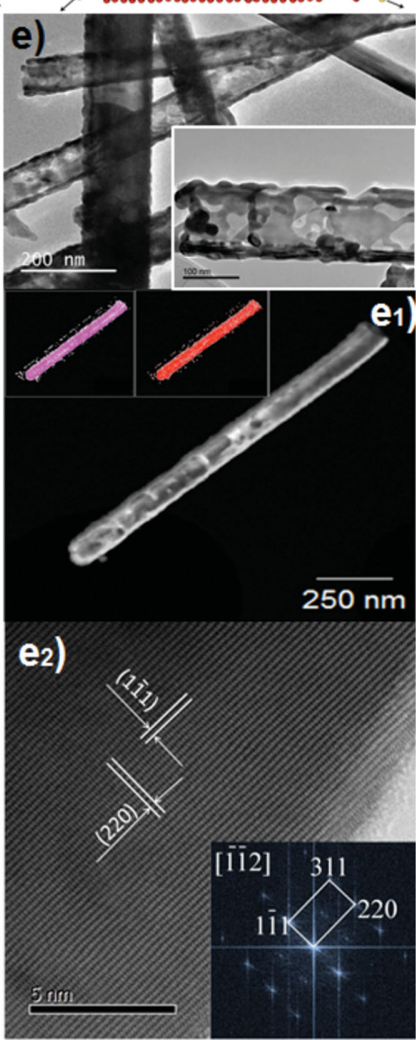

Figure 2. a) Schematic diagram of the formation of the nanotube electrocatalyst (yellow balls: Ag; red balls: Pd); HRTEM, EDS (pale red: Ag; red: Pd), and STEM images of b) Ag NWs b1, b2), c) AgPd-1 (c1, c2), d) AgPd-2 (d1, d2), and e) AgPd-3 (e1, e2). The insets in b2, c2, d2, and e2 are the corresponding fast Fourier transform patterns.

phase has formed on the surface of the NT. Therefore, a range of compositions of AgPd alloy and Pd were synthesized in this corrosion process. Some corroded holes were formed in the walls of the NTs, leading to the formation of porous AgPd-Pd NTs, as shown in the inset of Figure 2e. However, the NT structure will be damaged in further replacement reaction with increasing the amount of $\mathrm{Pd}^{2+}$ ion (Figure S2b, Supporting Information).

The rotating disk electrode (RDE) technique was used to investigate the ORR activities of the four samples including pure Ag NWs and the AgPd-1, AgPd-2, and AgPd-3 (AgPd-Pd) NTs compared with high-quality commercial Pt/C (20 wt\% Pt on Vulcan XC-72) in $\mathrm{O}_{2}$ saturated $0.1 \mathrm{~m} \mathrm{KOH}$ electrolyte at a scan rate of $10 \mathrm{mV} \mathrm{s}^{-1}$. As shown in the RDE curves in Figure 3a and Figure S3 (Supporting Information), compared with the Ag NWs and AgPd NTs, AgPd-3 features an enhanced ORR performance with positive onset potential and larger current density than AgPd-1 and AgPd-2. Furthermore, the RDE curves at various rotation speeds were measured to determine its ORR kinetic performance (details are given in the Supporting Information). The corresponding Koutecky-Levich $(\mathrm{K}-\mathrm{L})$ plots $\left(\mathrm{J}^{-1}\right.$ vs $\left.\omega^{-1 / 2}\right)$ at various electrode potentials exhibit good linearity (inset images in Figure S3, Supporting Information). The linearity and parallelism of the K-L plots suggest first-order reaction kinetics toward the concentration of dissolved oxygen and similar electron transfer numbers for the ORR at different potentials. The AgPd-3 NTs favor a nearly 4-electron ORR process. AgPd-1 and AgPd-2 also show a high electron reaction, corresponding to 3.6 and 3.7, respectively. The catalytic activity enhancement of the AgPd NTs over the commercial $\mathrm{Pt} / \mathrm{C}$ and pure $\mathrm{Ag} \mathrm{NWs}$ is plotted as a function of potential in Figure 3b and Figure S4 (Supporting Information). In Figure 3b, the AgPd NTs show large activity improvements over the commercial $\mathrm{Pt} / \mathrm{C}$, which are largely attributable to the AgPd alloy layer and the porous structure of the NTs. At $0.95 \mathrm{~V}$, the AgPd-3 NTs show an improvement over commercial Pt/C by a factor of nearly eight. Compared with Ag NWs, AgPd-3 NTs also show an improvement by a factor of nearly 19 at $\approx 0.85 \mathrm{~V}$. This result is also consistent with the conclusion reached by Stevenson's and Xia's groups that AgPd alloy could enhance the catalytic activity by several times compared to pure monometallic systems. ${ }^{[13,38,40]}$ Cyclic voltammograms (CVs) in $\mathrm{O}_{2}$-saturated $0.1 \mathrm{M} \mathrm{KOH}$ electrolyte were also used to reveal the ORR activity of the as-prepared samples in Figure 3c. In the CV for pure Ag NWs, characteristic oxidation peaks appear between 1.067 and $1.467 \mathrm{~V}$, corresponding to $\mathrm{Ag}_{2} \mathrm{O}$ monolayer, $\mathrm{AgOH}$ bulk, and $\mathrm{Ag}_{2} \mathrm{O}$ bulk formation, respectively. ${ }^{[44,45]}$ The reduction of silver oxide is observed to be reversible on the negative scan, at $1.087 \mathrm{~V}$. In the case of the AgPd- $1 \mathrm{NTs}$, the oxide 

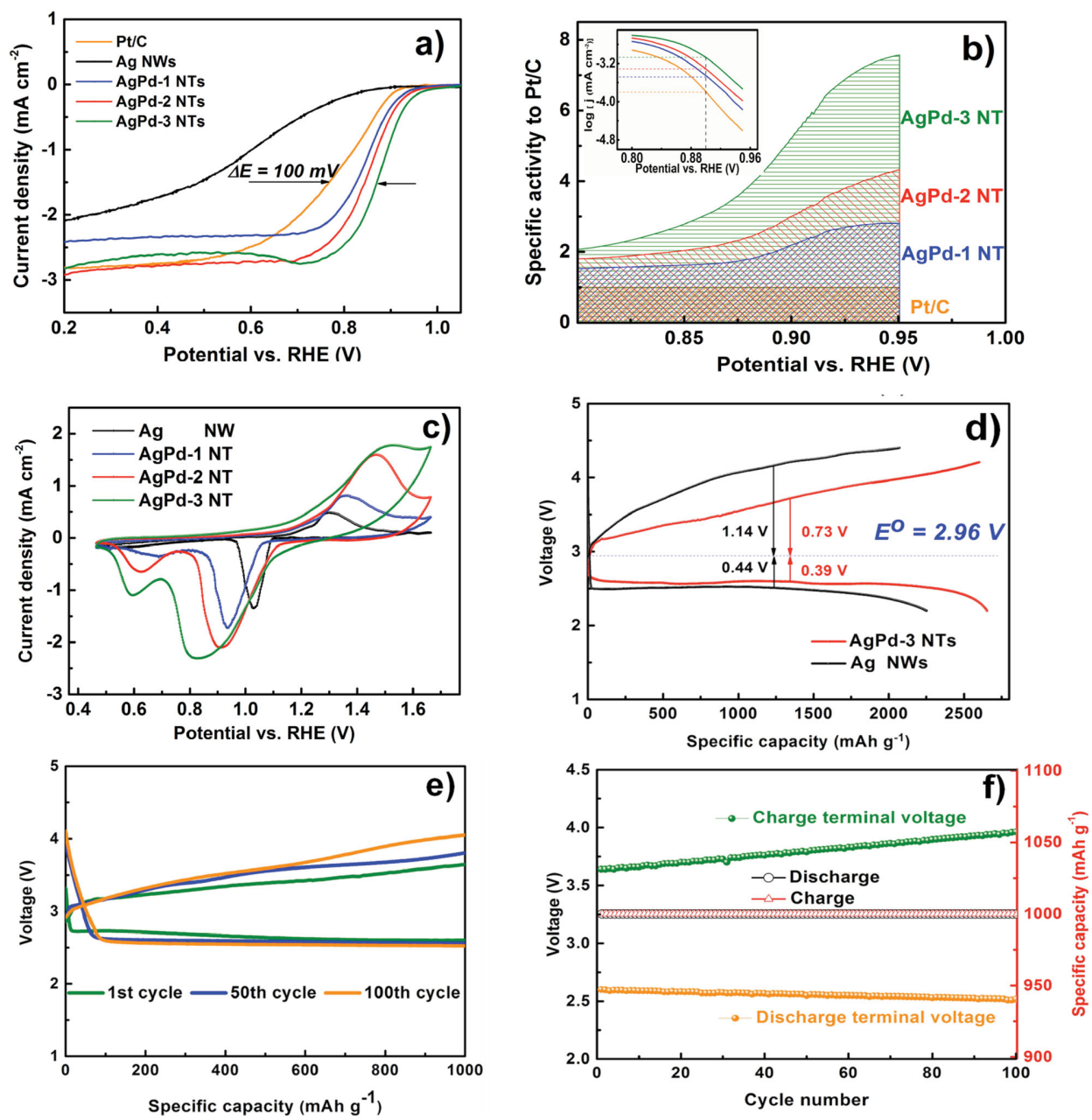

Figure 3. a) RDE curves of high-quality commercial Pt/C (20 wt\% Pt on Vulcan XC-72) and Ag NWs, AgPd-1, AgPd-2, AgPd-3 NTs; b) activity enhancement relative to commercial Pt/C $\left(\mathrm{j}_{\mathrm{k}} / \mathrm{j}_{\mathrm{k}}^{\mathrm{Ag}}\right)$, from the data shown in (a) (inset: Tafel slopes); c) cyclic voltammograms of Ag NWs, and AgPd-1, AgPd-2, $\mathrm{AgPd}-3 \mathrm{NTs}$; d) comparison of Ag NWs and AgPd-3 NTs on first discharge/charge results; e) discharge/charge curves of AgPd-3 NTs for selected cycles; f) cycling performance of the AgPd-3 NTs.

reduction peak of $\mathrm{Ag}$ is shifted left, which may be due to the alloy layer on the surface. The same trend in the shift continues with an increasing ratio of $\mathrm{Pd}$ in the composite. Combining all the above results, it is concluded that an AgPd alloy layer has formed on the surface and is essential for achieving enhanced catalytic performance.

The electrochemical properties of the samples were then examined in a lithium-oxygen cell without using any additional conductive carbon black. The details of the electrode preparation and cell assembly are provided in the Supporting Information. The specific capacities were calculated based on the total composite mass in the cathodes. The pure Ag NWs and AgPd-3 NTs are included for comparison in Figure 3d, which shows the first discharge/charge voltage profiles of the electrodes with pure Ag NWs and AgPd-3 NT composite at a current density of $0.2 \mathrm{~mA} \mathrm{~cm}{ }^{-2}$. Compared with the pure Ag NWs, the AgPd-3 NTs show improved round-trip efficiency up to $78 \%$, which is vital for electrochemical energy storage devices, together with a discharge capacity of $2650 \mathrm{~mA} \mathrm{~h} \mathrm{~g}^{-1}$ and a charge capacity of $2600 \mathrm{~mA} \mathrm{~h} \mathrm{~g}^{-1}$ at a current density of
$0.2 \mathrm{~mA} \mathrm{~cm}^{-2}$. Furthermore, the charge plateau of the AgPd-3 NTs is at $3.69 \mathrm{~V}$, lower than those of the Ag NWs and some previously reported results, ${ }^{[16,46-48]}$ showing the excellent OER performance. These results can also be further confirmed by the CVs and RDE curves in $\mathrm{O}_{2}$ saturated nonaqueous electrolyte $\left(1 \mathrm{M} \mathrm{LiCF}_{3} \mathrm{SO}_{3}\right.$ in tetraethylene glycol dimethyl ether (TEGDME)), as shown in Figure S5 (Supporting Information). Compared with Ag NWs, the AgPd-3 NTs display obviously higher ORR and OER currents and smaller overpotentials, which indicate that the AgPd-3 NTs provide a bifunctional catalyst performance in the anodic and cathodic scan processes. In organic electrolyte, AgPd-3 NTs also show positive onset potential and larger current density with increased rotating speed. In Figure S6 (Supporting Information), when the current density is increased to 0.4 and $0.6 \mathrm{~mA} \mathrm{~cm}^{-2}$, the AgPd-3 NTs also exhibit excellent discharge/charge performance, with 2275/1970 and $1770 / 1445 \mathrm{~mA} \mathrm{~h} \mathrm{~g}^{-1}$, respectively. More importantly, excellent energy efficiency of $65 \%\left(0.4 \mathrm{~mA} \mathrm{~cm}^{-2}\right)$ and $60 \%\left(0.6 \mathrm{~mA} \mathrm{~cm}{ }^{-2}\right)$ can also be achieved at higher current densities. All of this excellent performance can be attributed to the high catalytic 

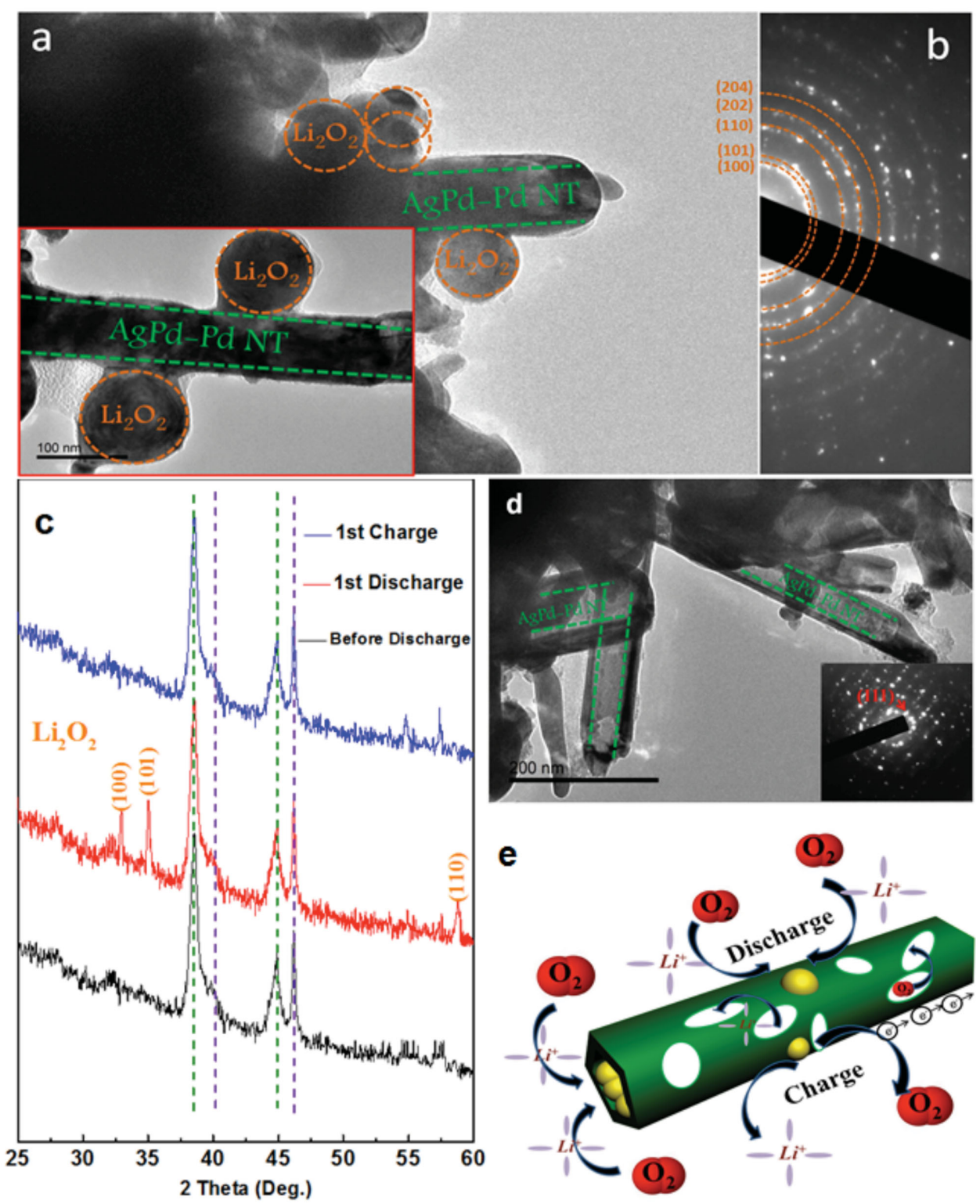

Figure 4. a) TEM image and b) SAED pattern of AgPd-Pd NT electrocatalyst after full discharge; c) XRD patterns of the AgPd-Pd NT electrocatalyst collected at different reaction steps; d) HRTEM image of AgPd-Pd NT electrocatalyst after the first cycle (corresponding SAED pattern as inset); e) schematic diagram of the discharge and charge processes.

activity of the AgPd-3 NTs and the porous NT structure, with porous channels facilitating rapid $\mathrm{O}_{2}$ diffusion and providing a high density of reactive sites. The capacity-limited method was used to evaluate the cycling performance. ${ }^{[15,49]}$ Figure 3e,f presents the discharge/charge and cycling performances of the AgPd-3 NT composite at $0.2 \mathrm{~mA} \mathrm{~cm}^{-2}$ current density with a fixed specific capacity of $1000 \mathrm{~mA} \mathrm{~h} \mathrm{~g}{ }^{-1}$. The AgPd-3 NTs show a high ORR potential and a low OER potential even after 100 cycles (Figure 3e). In Figure 3f, the discharge and charge capacities retain stable values, and, in addition, the discharge terminal voltage is higher than $2.5 \mathrm{~V}$, while the charge terminal potential is lower than $4.1 \mathrm{~V}$ for 100 cycles. Generally speaking, the AgPd-3 NTs exhibit superior electrochemical performance to those in other published results, ${ }^{[16,18,37,48,49]}$ resulting from both the efficient synergistic catalytic activity and the continuous $3 \mathrm{D}$ reaction network formed by the porous NTs.

To further understand the AgPd-Pd NT (AgPd-3 NTs) reaction mechanism over the whole process, the XRD patterns and HRTEM morphologies of the air electrode at different discharge/charge stages are shown in Figure 4. In the discharged samples, as shown in Figure 4a, the reaction produces $\mathrm{Li}_{2} \mathrm{O}_{2}$ nanocrystals (marked by orange circles) with particle size in the range of $50-100 \mathrm{~nm}$ that nucleate on the surface and inside of the NTs, which can be observed from the disappearance of the hollow structure in the inset of Figure 4a and Figure S7 (Supporting Information). The diffraction signal was also used to prove the presence of crystallized $\mathrm{Li}_{2} \mathrm{O}_{2}$, with five diffraction rings in the corresponding SAED pattern, as 
shown in Figure 4b. In previously reported results, very large toroid-like $\mathrm{Li}_{2} \mathrm{O}_{2}$ particles with size up to $1 \mu \mathrm{m}$ can normally be observed during the ORR process. ${ }^{[46,50]}$ What we have here is very different. The different morphology of $\mathrm{Li}_{2} \mathrm{O}_{2}$, which could be attributed to the different electrocatalyst and its morphology, could result in different electrochemical performance. ${ }^{[16,46,49-51]}$ After full charging, the obvious NT structure appears again (Figure $4 \mathrm{~d}$ ), indicating the high reversibility of $\mathrm{Li}_{2} \mathrm{O}_{2}$. The corresponding SAED pattern in the inset of Figure 4d confirms the cubic structure of the AgPd and Pd composite. The XRD patterns in Figure 4c also confirm the reversible formation of $\mathrm{Li}_{2} \mathrm{O}_{2}$ during the discharge and charge processes, which is consistent with the results obtained by TEM observation and other groups. ${ }^{[46,47,51,52]}$ The diagram in Figure 4e schematically outlines the discharge and charge processes. The NT structure with porous channels, facilitating rapid $\mathrm{O}_{2}$ and electrolyte diffusion, forms a continuous conductive network throughout the whole ORR and OER process. It also can provide a high density of reactive sites on the outside and inside of the NT, in which $\mathrm{Li}_{2} \mathrm{O}_{2}$ can be deposited to achieve high energy density. In addition, the electrolyte stability was also investigated by FTIR and Raman spectra during long-term capacity-limited cycling. According to the published results, ether-based electrolyte is prone to autoxidation under oxygenated radicals and occurs decomposition higher than $4 \mathrm{~V}$, leading to formation of nonreversible reaction products. ${ }^{[53,54]}$ As shown in Figure S8 (Supporting Information), although TEGDME is more stable than other organic solvent, such as carbonate-based and other ether-based electrolyte, electrolyte decomposition also appears after 65 cycles giving a mixture of nonreversible reaction products, such as $\mathrm{Li}_{2} \mathrm{CO}_{3}, \mathrm{LiOH}$, which increase the overpotential and influence the cycling performance. X-ray photoelectron spectroscopy (XPS) was further used to gain insight into the Ag and Pd chemical bonding information during long-term cycling as shown in Figure S9 (Supporting Information). Compared with the aqueous system, Ag and Pd are much more stable in $\mathrm{O}_{2}$ saturated TEGDME electrolyte. Unfortunately, after long-term cycling, accompanied by the electrolyte decomposition, $\mathrm{Ag}$ could be transferred into $\mathrm{Ag}^{+}$ion or $\mathrm{Ag}_{2} \mathrm{O}$ because of adsorption of $\mathrm{OH}^{-}$ions followed by further oxidation at more positive potentials.

In conclusion, carbon-free porous AgPd-Pd composite NTs were synthesized via a galvanic replacement reaction. The composite was used as a bifunctional electrocatalyst, showing favorable rechargeability and good electrocatalyst performance with a high round-trip efficiency, owing to the high catalytic activity of the electrocatalyst and the abundant catalytic active sites provided by the porous NT structure. Therefore, this AgPd-Pd electrocatalyst with carbon-free porous NT structure is a promising bifunctional electrocatalyst for lithium-oxygen batteries, with high energy density, favorable rechargeability, and high round-trip performance.

\section{Experimental Section}

Synthesis of Ag Nanowires: Ag NWs were synthesized by reducing $\mathrm{AgNO}_{3}$ with ethylene glycol (EG, Sigma) in the presence of Pt seeds and poly(vinyl pyrrolidone) (PVP) $\left(M_{\mathrm{w}}=40000\right)$. In a typical synthesis, $50 \mathrm{~mL}$ EG was added to a $100 \mathrm{~mL}$ round flask. It was then partially immersed in an oil bath and kept at $165^{\circ} \mathrm{C}$ for 10 min under ambient pressure. In the second step, $5 \mathrm{~mL} \mathrm{H}_{2} \mathrm{PtCl}_{6}$ solution $\left(2 \times 10^{-4}\right.$, in $\left.\mathrm{EG}\right)$ was added. After $10 \mathrm{~min}, 25 \mathrm{~mL} \mathrm{AgNO}$ solution $(0.12 \mathrm{M}$, in EG) and $50 \mathrm{~mL}$ PVP solution $(0.36 \mathrm{M}$, in $\mathrm{EG})$ were added dropwise to the hot solution over a period of $10 \mathrm{~min}$. The reaction continued at $165^{\circ} \mathrm{C}$ for $60 \mathrm{~min}$ with an air cooling reflux system. Vigorous stirring was maintained throughout the entire process. The obtained composite was collected and aged in $\mathrm{NH}_{3} \cdot \mathrm{H}_{2} \mathrm{O}$ for $1 \mathrm{~d}$ to remove the $\mathrm{AgCl}$. Finally, the reaction mixture was diluted with water and acetone followed by centrifuging at $3000 \mathrm{rpm}$ for $15 \mathrm{~min}$. The supernatant containing silver particles could then be easily removed using a pipette. This centrifugation procedure was repeated several times until the supernatant became colorless.

Synthesis of Electrocatalyst Nanotubes: $10 \mathrm{~mL}$ of the obtained Ag NW/s was diluted with $100 \mathrm{~mL}$ water and then refluxed in a three-necked round bottom flask at boiling temperature for $10 \mathrm{~min}$. $50 \mathrm{~mL} \mathrm{PdCl}$ solution with different concentrations for different samples was added dropwise to the solution over $15 \mathrm{~min}$. This mixture was continuously refluxed until its color became stable. Finally, the obtained composite (AgPd-1, AgPd-2, and AgPd-3 NTs) reaction mixture was collected and aged in $\mathrm{NH}_{3} \cdot \mathrm{H}_{2} \mathrm{O}$ for $2 \mathrm{~d}$ to remove the $\mathrm{AgCl}$. The obtained composites were further washed with water and acetone several times. Before the composites were used as catalyst, acid treatment and annealing treatment were needed. The acid treatment was performed in $0.5 \mathrm{M} \mathrm{HNO}_{3}$ solution for $2 \mathrm{~h}$ under stirring. The heat treatment was carried out under flowing argon gas in an oven at $250^{\circ} \mathrm{C}$ for $2 \mathrm{~h}$.

Characterization: The structure and phase purity were analyzed by X-ray powder diffraction (GBC MMA) on an instrument equipped with $\mathrm{Cu} \mathrm{K} \alpha$ radiation that was operated over a $2 \theta$ range of $30^{\circ}-80^{\circ}$ in continuous scan mode with a scan rate of $0.5^{\circ} \mathrm{min}^{-1}$. Refinement results were calculated using GASAII software. The morphology of the samples was examined using FESEM (JEOL JSM-7500). Transmission electron microscopy (TEM) investigations were performed using a $200 \mathrm{kV} \mathrm{JEOL} 2011$ instrument. Atomic resolution analytical microscope investigations were performed using a $200 \mathrm{kV}$ JEOL 2011 instrument. Raman spectroscopy was performed using a Raman spectrometer (Jobin Yvon HR800) employing a $10 \mathrm{~mW}$ neon laser at $632.8 \mathrm{~nm}$. FTIR spectra were collected using an FTIR Prestige-21 (Shimadzu). XPS experiments were carried out on a VG Scientific ESCALAB 2201XL instrument using aluminum $\mathrm{K} \alpha \mathrm{X}$-ray radiation. XPS spectral analysis was conducted using XPS Peak-fit software.

Catalyst and Electrochemical Performance: Electrochemical performance was tested using CR2032 type coin cells with holes in the cathode parts. To prepare the working electrodes, a mixture of the as-synthesized catalyst and poly(tetrafluoroethylene) in a weight ratio of $90: 10$, using Nafion (5\%) as the solvent, was pasted onto the stainless steel mesh (mesh edge was welded on the cathode shell). Typical loadings of cathode powder for $1.1 \mathrm{~cm}^{2}$ were $\approx 2 \mathrm{mg} \mathrm{cm}^{-2}$. The electrolyte consisted of a solution of $1 \mathrm{M} \mathrm{LiCF} \mathrm{SO}_{3}$ in TEGDME. Pure lithium foil was used as the counter electrode. The cells were assembled in an argon-filled glove-box (Mbraun, Unilab, Germany). Galvanostatic deep, full charge-discharge curves were collected at various current densities of $0.2,0.4$, and $0.6 \mathrm{~mA} \mathrm{~cm}^{-2}$ between 4.2 and $2.5 \mathrm{~V}$ versus $\mathrm{Li}^{+} / \mathrm{Li}$, and discharge-charge cycling was conducted with $1000 \mathrm{~mA} \mathrm{~h} \mathrm{~g}^{-1}$ cutoff discharge and charge capacity. All tests were conducted with LAND CT 2001A multichannel battery testers at room temperature in oxygen atmosphere, using our designed facility. RDE aqueous electrochemical tests were carried out using a computer-controlled potentiostat (Princeton 2273 and 616, Princeton Applied Research) with a typical three-electrode cell. The details of assembling the testing system and electrode preparation can be found in our reported work. ${ }^{[16]}$ The working electrodes were prepared by using the respective catalyst inks on the prepolished glassy carbon disk electrodes. Platinum foil was used as the counter-electrode and an $\mathrm{Ag} / \mathrm{AgCl}$ (saturated $\mathrm{KCl}$ filled) electrode was used as the reference electrode. The detailed kinetic analysis was conducted according to $\mathrm{K}-\mathrm{L}$ plots:

$\frac{1}{j}=\frac{1}{j_{k}}+\frac{1}{B \omega^{0.5}}$ 
In equation (1), $j_{k}$ is the kinetic current and $B$ is the Levich slope, which is given by:

$B=0.2 n F\left(D_{\mathrm{O}_{2}}\right)^{2 / 3} v^{-1 / 6} C_{\mathrm{O}_{2}}$

Here, $n$ is the number of electrons transferred in the reduction of one $\mathrm{O}_{2}$ molecule, $F$ is the Faraday constant $\left(F=96485 \mathrm{C} \mathrm{mol}^{-1}\right), D_{\mathrm{O} 2}$ is the diffusion coefficient of $\mathrm{O}_{2}\left(D_{\mathrm{O} 2}=1.9 \times 10^{-5} \mathrm{~cm}^{2} \mathrm{~s}^{-1}\right), v$ is the kinematic viscosity for $\mathrm{KOH}\left(\nu=0.01 \mathrm{~cm}^{2} \mathrm{~s}^{-1}\right)$, and $\mathrm{C}_{\mathrm{O} 2}$ is the concentration of $\mathrm{O}_{2}$ in the solution $\left(C_{\mathrm{O} 2}=1.2 \times 10^{-6} \mathrm{~mol} \mathrm{~cm}^{-3}\right)$. The constant 0.2 is adopted when the rotation speed is expressed in rpm. According to Equation (1) and (2), the number of electrons transferred ( $n$ ) can be obtained from the slope of the $\mathrm{K}-\mathrm{L}$ plot of $j^{-1}$ versus $\omega^{-1 / 2}$. CVs were also obtained using the same procedure.

The nonaqueous electrochemical cyclic voltammetry (CV) tests were carried out using computer-controlled potentiostats (Princeton 2273 and 636, Princeton Applied Research) in a three-electrode system. $1 \mathrm{M}$ $\mathrm{LiCF}_{3} \mathrm{SO}_{3}$ in TEGDME was used as electrolyte. The details of assembling the testing system and electrode preparation can be found in our reported work. ${ }^{[13]} \mathrm{CVs}$ were obtained from open voltage to $3.6 \mathrm{~V}$. The above procedure was repeated for each sample.

\section{Supporting Information}

Supporting Information is available from the Wiley Online Library or from the author.

\section{Acknowledgements}

The manuscript was written through contributions of all authors. All authors have given approval to the final version of the manuscript. This work was supported by the Australian Research Council (ARC) through a Discovery Project (DP140100401) and a Linkage Project (LP120200432). The authors would like to also thank Dr. Tania Silver for critical reading of the manuscript, and also acknowledge the use of the facilities in the UOW Electron Microscopy Center, with particular thanks to Dr. Gilberto Casillas-Garcia.

Received: May 11, 2015

Revised: June 26, 2015

Published online: September 24, 2015

[1] K. Otsubo, Y. Wakabayashi, J. Ohara, S. Yamamoto, H. Matsuzaki, H. Okamoto, K. Nitta, T. Uruga, H. Kitagawa, Nat. Mater. 2011, 10, 291.

[2] N. Du, H. Zhang, B. D. Chen, X. Y. Ma, Z. H. Liu, J. B. Wu, D. R. Yang, Adv. Mater. 2007, 19, 1641.

[3] K. X. Wang, M. D. Wei, M. A. Morris, H. S. Zhou, J. D. Holmes, Adv. Mater. 2007, 19, 3016.

[4] J. Goldberger, R. R. He, Y. F. Zhang, S. W. Lee, H. Q. Yan, H. J. Choi, P. D. Yang, Nature 2003, 422, 599.

[5] A. L. M. Reddy, M. M. Shaijumon, S. R. Gowda, P. M. Ajayan, Nano Lett. 2009, 9, 1002.

[6] R. Cai, H. Liu, W. Y. Zhang, H. T. Tan, D. Yang, Y. Z. Huang, H. H. Hng, T. M. Lim, Q. Y. Yan, Small 2013, 9, 1036.

[7] Y. Zhao, W. L. Wu, J. X. Li, Z. C. Xu, L. H. Guan, Adv. Mater. 2014, 26, 5113.

[8] S. J. Guo, X. Zhang, W. L. Zhu, K. He, D. Su, A. MendozaGarcia, S. F. Ho, G. Lu, S. H. Sun, J. Am. Chem. Soc. 2014, 136, 15026.
[9] J. S. Jie, C. Y. Wu, Y. Q. Yu, L. Wang, Z. Z. Hu, Nanotechnology 2009, 20,455702 .

[10] R. Cai, J. Chen, J. X. Zhu, C. Xu, W. Y. Zhang, C. M. Zhang, W. H. Shi, H. T. Tan, D. Yang, H. H. Hng, T. M. Lim, Q. Y. Yan, J. Phys. Chem. C 2012, 116, 12468.

[11] N. Du, H. Zhang, B. Chen, J. B. Wu, X. Y. Ma, Z. H. Liu, Y. Q. Zhang, D. Yang, X. H. Huang, J. P. Tu, Adv. Mater. 2007, 19, 4505.

[12] C. Liu, F. Li, L. P. Ma, H. M. Cheng, Adv. Mater. 2010, 22, E28.

[13] Y. N. Xia, P. D. Yang, Y. G. Sun, Y. Y. Wu, B. Mayers, B. Gates, Y. D. Yin, F. Kim, Y. Q. Yan, Adv. Mater. 2003, 15, 353.

[14] T. Yang, B. A. Lu, Phys. Chem. Chem. Phys. 2014, 16, 4115.

[15] J. Lu, Y. Lei, K. C. Lau, X. Y. Luo, P. Du, J. G. Wen, R. S. Assary, U. Das, D. J. Miller, J. W. Elam, H. M. Albishri, D. Abd El-Hady, Y. K. Sun, L. A. Curtiss, K. Amine, Nat. Commun. 2013, 4, 1

[16] W. B. Luo, S. L. Chou, J. Z. Wang, Y. C. Zhai, H. K. Liu, Small 2015, $10,1002$.

[17] M. Park, H. Sun, H. Lee, J. Lee, J. Cho, Adv. Energy Mater. 2012, 2, 780.

[18] Z. Q. Peng, S. A. Freunberger, Y. H. Chen, P. G. Bruce, Science 2012, 337, 563.

[19] Y. Y. Shao, F. Ding, J. Xiao, J. Zhang, W. Xu, S. Park, J. G. Zhang, Y. Wang, J. Liu, Adv. Funct. Mater. 2013, 23, 987.

[20] J. Xiao, D. H. Mei, X. L. Li, W. Xu, D. Y. Wang, G. L. Graff, W. D. Bennett, Z. M. Nie, L. V. Saraf, I. A. Aksay, J. Liu, J. G. Zhang, Nano Lett. 2011, 11, 5071.

[21] W. Hong, J. Wang, E. K. Wang, Small 2014, 10, 3262.

[22] C. Koenigsmann, S. S. Wong, Energy Environ. Sci. 2011, 4, 1161.

[23] B. Lim, M. J. Jiang, P. H. C. Camargo, E. C. Cho, J. Tao, X. M. Lu, Y. M. Zhu, Y. N. Xia, Science 2009, 324, 1302.

[24] M. H. Oh, T. Yu, S. H. Yu, B. Lim, K. T. Ko, M. G. Willinger, D. H. Seo, B. H. Kim, M. G. Cho, J. H. Park, K. Kang, Y. E. Sung, N. Pinna, T. Hyeon, Science 2013, 340, 964.

[25] Y. G. Sun, Y. Ren, Y. Z. Liu, J. G. Wen, J. S. Okasinski, D. J. Miller, Nat. Commun. 2012, 3, 971.

[26] X. H. Xia, Y. Wang, A. Ruditskiy, Y. N. Xia, Adv. Mater. 2013, 25, 6313.

[27] Y. G. Sun, B. Mayers, Y. N. Xia, Adv. Mater. 2003, 15, 641.

[28] Y. Y. Jia, Y. Q. Jiang, J. W. Zhang, L. Zhang, Q. L. Chen, Z. X. Xie, L. S. Zheng, J. Am. Chem. Soc. 2014, 136, 3748.

[29] P. Liu, J. K. Norskov, Phys. Chem. Chem. Phys. 2001, 3, 3814.

[30] M. Mavrikakis, B. Hammer, J. K. Norskov, Phys. Rev. Lett. 1998, 81, 2819.

[31] J. A. Rodriguez, M. Kuhn, J. Hrbek, J. Phys. Chem. 1996, 100, 15494.

[32] X. L. Sun, D. G. Li, Y. Ding, W. L. Zhu, S. J. Guo, Z. L. Wang, S. H. Sun, J. Am. Chem. Soc. 2014, 136, 5745.

[33] Y. E. Wu, D. S. Wang, G. Zhou, R. Yu, C. Chen, Y. D. Li, J. Am. Chem. Soc. 2014, 136, 11594.

[34] S. F. Xie, S. I. Choi, N. Lu, L. T. Roling, J. A. Herron, L. Zhang, J. Park, J. G. Wang, M. J. Kim, Z. X. Xie, M. Mavrikakis, Y. N. Xia, Nano Lett. 2014, 14, 3570.

[35] S. Zhang, Y. Z. Hao, D. Su, V. V. T. Doan-Nguyen, Y. T. Wu, J. Li, S. H. Sun, C. B. Murray, J. Am. Chem. Soc. 2014, 136, 15921

[36] S. J. Guo, S. Zhang, D. Su, S. H. Sun, J. Am. Chem. Soc. 2013, 135, 13879.

[37] Y. C. Lu, Z. C. Xu, H. A. Gasteiger, S. Chen, K. Hamad-Schifferli, Y. Shao-Horn, J. Am. Chem. Soc. 2010, 132, 12170.

[38] D. A. Slanac, W. G. Hardin, K. P. Johnston, K. J. Stevenson, J. Am. Chem. Soc. 2012, 134, 9812.

[39] N. L. Zhang, X. Chen, Y. J. Lu, L. An, X. Li, D. G. Xia, Z. Zhang, J. X. Li, Small 2014, 10, 2662.

[40] Y. G. Sun, Z. L. Tao, J. Chen, T. Herricks, Y. N. Xia, J. Am. Chem. Soc. 2004, 126, 5940

[41] M. H. Shao, T. Huang, P. Liu, J. Zhang, K. Sasaki, M. B. Vukmirovic, R. R. Adzic, Langmuir 2006, 22, 10409 
[42] J. Reyes-Gasga, J. L. Elechiguerra, C. Liu, A. Camacho-Bragado, J. M. Montejano-Carrizales, M. J. Yacaman, J. Cryst. Growth 2006, 286, 162.

[43] C. Damle, A. Kumar, M. Sastry, J. Phys. Chem. B 2002, 106, 297.

[44] J. S. Guo, A. Hsu, D. Chu, R. R. Chen, J. Phys. Chem. C 2010, 114, 4324.

[45] M. Hepel, M. Tomkiewicz, J. Electrochem. Soc. 1984, 131, 1288.

[46] R. R. Mitchell, B. M. Gallant, C. V. Thompson, Y. Shao-Horn, Energy Environ. Sci. 2011, 4, 2952.

[47] E. Yilmaz, C. Yogi, K. Yamanaka, T. Ohta, H. R. Byon, Nano Lett. 2013, 13, 4679 .

[48] L. X. Zhang, S. L. Zhang, K. J. Zhang, G. J. Xu, X. He, S. M. Dong, Z. H. Liu, C. S. Huang, L. Gu, G. L. Cui, Chem. Commun. 2013, 49 3540 .
[49] Z. L. Jian, P. Liu, F. J. Li, P. He, X. W. Guo, M. W. Chen, H. S. Zhou, Angew. Chem. Int. Ed. 2014, 53, 442.

[50] M. D. Bhatt, H. Geaney, M. Nolan, C. O'Dwyer, Phys. Chem. Chem. Phys. 2014, 16, 12093.

[51] R. R. Mitchell, B. M. Gallant, Y. Shao-Horn, C. V. Thompson, J. Phys. Chem. Lett. 2013, 4, 1060.

[52] Y. G. Wang, Y. Y. Xia, Nat. Chem. 2013, 5, 445.

[53] B. D. McCloskey, R. Scheffler, A. Speidel, D. S. Bethune, R. M. Shelby, A. C. Luntz, J. Am. Chem. Soc. 2011, 133, 18038.

[54] S. A. Freunberger, Y. H. Chen, N. E. Drewett, L. J. Hardwick, F. Barde, P. G. Bruce, Angew. Chem. Int. Ed. 2011, 50, 8609. 